\title{
A Meta-Analysis in Assessing Oxidative Stress Using Malondialdehyde in Oral Submucous Fibrosis
}

\author{
Khadijah Mohideen ${ }^{1} \quad$ Chandrasekaran Krithika ${ }^{2} \quad$ Nadeem Jeddy ${ }^{1} \quad$ Sameena Parveen ${ }^{3}$ Thangavelu Radhika ${ }^{1}$ \\ S. Leena Sankari ${ }^{4}$
}

${ }^{1}$ Department of Oral Pathology and Microbiology, Dr. M.G.R. Educational and Research Institute, Thai Moogambigai Dental College and Hospital, Chennai, Tamil Nadu, India

2Department of Oral Medicine and Radiology, Dr. M.G.R. Educational and Research Institute, Thai Moogambigai Dental College and Hospital, Chennai, Tamil Nadu, India

${ }^{3}$ Department of Maxillofacial Surgery and Diagnostic Sciences, College of Dentistry, Jazan University, Jazan, Saudi Arabia

${ }^{4}$ Department of Oral Pathology and Microbiology, Bharath Institute of Higher Education and Research, Sree Balaji Dental College and Hospital, Chennai, Tamil Nadu, India

\begin{abstract}
Address for correspondence Khadijah Mohideen, MDS, Research Scholar, Department of Oral Pathology and Microbiology, Dr. M.G.R. Educational and Research Institute, Thai Moogambigai Dental College and Hospital, Maduravoyal, Chennai 600095, Tamil Nadu, India (e-mail: dr.khadijahm@gmail.com).
\end{abstract}

Eur J Dent 2021;15:675-681.

Abstract
Keywords
- oral submucous
fibrosis
- potentially malignant
disorders
- precancer
- oxidative stress

Objective This study aimed to evaluate the oxidative stress (OS) marker Malondialdehyde (MDA) in oral submucous fibrosis with available literature.

Materials and Methods We conducted a literature search electronically in PubMed (MeSH), Science Direct, Scopus, and Google Scholar using specific keywords.

Results A systematic search in PubMed, Science Direct, and Google Scholar identified 334 articles. Of these, four were duplicate reports, and three were animal studies. After reading the abstracts of the collected articles, 288 articles were excluded for the following reasons: low quality, not relevant to the research question, or did not meet the inclusion criteria. The remaining 46 articles were chosen for full-text assessment. Finally, the present qualitative synthesis included 23 articles for evaluation. The selected studies in MDA analysis in a random-effects model showed higher heterogeneity $\left(Q=477.636, p<0.001, I^{2}=95.394 \%\right)$. The standard difference in mean MDA concentration between oral submucous fibrosis (OSMF) and healthy subjects was estimated as $2.73 \mathrm{nmol} / \mathrm{mL}$ (95\% confidence interval: $2.08-3.38$ ).

Conclusion The selected studies showed significantly higher MDA levels in various biological samples of patients with OSMF. Therefore, further studies are needed to estimate oxidative stress levels by using different biomarkers in OSMF to direct future therapy.

\section{Introduction}

Oral submucous fibrosis (OSMF) is a chronic, insidious, and progressive disease characterized by fibro-elastic changes in the mucosa that leads to trismus. OSMF is one of the most frequently occurring, potentially malignant disorders of South-East Asian descent. ${ }^{1}$ It has also been frequently reported in Europe and North America. ${ }^{2}$ In India, the reported incidence rate of OSMF is four per 1,000 adults. About 5 million young Indians suffer from OSMF. ${ }^{3}$ The disease is multifactorial in origin. Tobacco smoking and areca
Dol https://doi.org/ $10.1055 / \mathrm{s}-0041-1728230$ ISSN $1305-7456$ (c) 2021. European Journal of Dentistry.

This is an open access article published by Thieme under the terms of the Creative Commons Attribution-NonDerivative-NonCommercial-License, permitting copying and reproduction so long as the original work is given appropriate credit. Contents may not be used for commercial purposes, or adapted, remixed, transformed or built upon. (https://creativecommons.org/licenses/by-nc-nd/4.0/)

Thieme Medical and Scientific Publishers Pvt. Ltd., A-12, 2nd Floor, Sector 2, Noida-201301 UP, India 
nut consumption are the primary causatives for oral submucous fibrosis. ${ }^{4} \mathrm{~A}$ recent rise in the incidence of this disorder, especially in the younger group, was reported in the literature even after a short period of betel quid chewing. The other factors responsible for the pathogenesis of OSMF include ingestion of chilies, deficient nutrition, genetic contribution, altered salivary constituents, autoimmunity, and collagen disorders. ${ }^{5}$ Current evidence supports collagen-related genes' role in the susceptibility and pathogenesis of OSMF. ${ }^{6}$

Oxidative tissue damage and lower defense by antioxidant enzymes could also be one of the causes. According to the literature, the biological matrix is continuously under oxidative stress (OS). Oxidative stress is a state that introduces a high production of pro-oxidants or free radicals and a low level of antioxidants. ${ }^{7}$ Pro-oxidants are highly reactive oxygen species (ROS). ${ }^{8}$ Various ROS in our body is derived from oxygen or nitrogen. ${ }^{9}$ They are intermediate molecules and by-products formed due to a disturbance in the various biological cycles. Age and genetics cause adverse changes in free radicals' production. ${ }^{10}$ Free radicals arise from the exogenous factors (e.g., X-ray, ozone exposure, tobacco smoking, pollutants, pan chewing, and various industrial chemicals) or from the endogenous factors of the normal metabolic process. ${ }^{11}$ The endogenous factors are mitochondrial reactions, xanthine oxidase activity, inflammation, phagocytosis, cyclooxygenase pathways, exercise, and ischemia-reperfusion injuries. ${ }^{12}$

The areca nut's phenolic compounds cause local injury and release inflammatory mediators, ROS, and cytokines. ROS reaction with cellular molecules forms DNA adducts, activates oncogenes, and leads to the inactivation of tumor suppressor genes. ${ }^{13}$ ROS reaction with biological molecules results in membrane lipid peroxidation and protein modification. The altered molecules affect gene expression and thereby promotes mutagenesis and carcinogenesis. ${ }^{14}$

Several compounds and enzymes may function to protect cellular components from oxidative damages of ROS and OS, which are known as antioxidants. ${ }^{15}$ These antioxidants play different vital roles like a radical scavenger, an enzyme inhibitor, hydrogen or electron donor, peroxide decomposer, or a metal chelating agent. ${ }^{16}$ Enzymes such as superoxide dismutase, catalase, glutathione peroxidase, and glutathione reductase exhibit antioxidant activity. Other compounds that serve as antioxidants are vitamins $\mathrm{A}, \mathrm{C}$, and E; minerals such as $\mathrm{Se}, \mathrm{Cu}$, $\mathrm{Mn}$, and $\mathrm{Zn}$; glutathione; flavonoids; bilirubin; and uric acid. ${ }^{9}$

In a healthy human, adequate balance is maintained between oxidants and antioxidants. A shift in the ratio toward pro-oxidants gives rise to OS. ${ }^{17}$ The excessive and frequent areca nut ingestion and tobacco exposure accelerate ROS production. Antioxidant enzymes counter the formed free radicals resulting in deprivation in antioxidant levels. ${ }^{18}$ Thus, the imbalance resulting from excessive ROS production by oxidative phosphorylation and suppression of antioxidant capacity generates OS, which may initiate and propagate fibrosis of the oral mucosa. Besides which also increases cytotoxicity and the chances of malignant transformation of potentially malignant disorders. ${ }^{19,20}$ The rate of malignant transformation of OSMF into oral squamous cell carcinoma is approximately estimated as $7.6 \%{ }^{2}$ Thus, the OS in the target cells and tissues has been suggested to play an essential role in progression of oral submucous fibrosis. ${ }^{21}$

Lipid peroxidation of polyunsaturated omega- 6 acyl group fatty acids by free radicals elevate the level of Malondialdehyde (MDA). ${ }^{22,23}$ Hence, the estimation of lipid peroxidation marker MDA in oral submucous fibrosis may serve as a biomarker to analyze the OS and disease progression.

\section{Materials and Methods}

\section{Electronic Search Identification}

We searched the electronic databases, including PubMed (MeSH), Science Direct, Scopus, and Google Scholar, for previously published articles that addressed the OS in oral submucous fibrosis using MDA levels between the years 2000 and 2020. We selected works only in English, using the following keywords such as oral submucous fibrosis, oxidative stress, and malondialdehyde.

\section{Screening for Relevance}

We identified the articles that discussed OS in OSMF. We shortlisted the titles and abstracts of all the collected materials for the screening of relevance and duplication.

\section{Exclusion Criteria}

Articles with unmatched objective and abstract:

- Being literature reviews and systematic reviews

- The studies included OSMF within the premalignant group without specific data for OSMF

- Studies used other OS markers and antioxidant enzymes or micronutrients as a marker of evaluation

- The works provided inadequate data for the comparison between control and OSMF groups

\section{Retrieval of Full-Text Articles and Evaluation}

Three observers independently evaluated all the presentations against the following criteria: selection bias, missing data or incomplete data, specification of data, imprecision (e.g., small sample size), quality measures (e.g., ethics approval, funding, and conflicts of interest statement) and other limitations. After assessing all the particulars, we have considered the articles for eligibility criteria.

\section{Inclusion Criteria}

- Studies discussed the oxidative status of OSMF using lipid peroxidation marker MDA

- Studies involving various biological samples and expressed the MDA data in mean, standard deviation along with $p$-value

- Papers provided sufficient data to allow comparison of OSMF and control groups 


\section{Data Extraction}

The extracted data from full-text articles were author, publication year, age groups, sample size, MDA measurements in OSMF, and control group expressed as the mean and standard deviation along with specific units. We tabulated all the collected data separately in a specified format (-Table $\mathbf{1}$ ). The statistical analysis was performed by comprehensive meta-analysis software for windows.

\section{Statistical Analysis}

The Forest plot was derived by using the standard difference in the mean method to carry out a meta-analysis using comprehensive meta-analysis software version 3 (Biostat Inc.; Englewood, New Jersey, United States). The standard difference in the mean value of MDA in OSMF was analyzed at a $95 \%$ confidence interval $(\mathrm{CI})$. The random-effects model was used in the analysis due to the presence of significant heterogeneity.

\section{Results}

From the methodology used, we retrieved 334 articles. PubMed search yielded 193 papers, Science Direct search yielded 35 papers, and Google Scholar search yielded 106 papers. After search refinement, 281 articles had unmatched titles and abstracts, four duplicated data reports and three articles were animal studies. After extraction of these articles, 46 articles had their titles relevant to the present work. Again we excluded the articles with unmatched objectives ( $n=13)$, systematic reviews $(n=2)$, and reviews $(n=3)$. We recovered 28 full-text articles with matching objectives. In the refined evaluation, we excepted the articles had not provided adequate data for comparison $(n=3)$. Therefore, we included only 25 articles, which forms the basis for the present work (-Fig. $\mathbf{1})$.

Of the 25 research articles, 23 articles were appropriate to the study of MDA in OSMF cases. In total, 23 included studies of MDA analysis in a random-effects model showed higher heterogeneity $\left(\mathrm{Q}=477.636, p<0.001, \mathrm{I}^{2}=95.394 \%\right)$. The standard difference in mean MDA concentration between OSMF and healthy subjects was estimated as $2.73 \mathrm{nmol} / \mathrm{mL}$ (95\% CI: 2.08-3.38; - Fig. 2).

\section{Publication Bias}

The studies' quality is considered by using the NewcastleOttawa quality assessment scale, as shown in - Table $\mathbf{1}$. Examining the funnel plot of precision by the standard difference in mean of studies included in the MDA estimation meta-analysis displayed a certain asymmetry, as shown in - Fig. 3. The heterogeneity is exposed by the high $I^{2}$ value (95.394\%).

\section{Discussion}

ROS-mediated lipid peroxidation leads to changes in the functional and structural integrity of the cell membrane. The malondialdehyde, a lipid peroxidation product, plays an essential role in promoting the malignant transformation. ${ }^{24}$ MDA helps collagen cross-linking by providing its aldehyde group to lysine and helps in lysine to lysine bridging in the presence of enzyme lysyl oxidase. The MDA-collagen cross-link complex will still contain a free reactive aldehyde group capable of reacting to different intermolecular cross-links. Hence, MDA facilitates inter- and intramolecular collagen cross-linking that stiffens the tissues and reduces their function. ${ }^{25}$ Levels of malondialdehyde have been recently correlated with clinical grades of oral submucous fibrosis. ${ }^{26}$ The altered states are expressed in various biological components like serum, plasma, tissue, and saliva. ${ }^{27}$ Thus, MDA assessment can be used as a reliable marker to assess tissue damage in pathological conditions such as OSMF. Hence, the present meta-analysis is considered to evaluate literature to analyze lipid peroxidation product (MDA) in various samples of patients diagnosed with oral submucous fibrosis and to compare with the healthy subjects.

The comprehensive meta-analysis of research articles in the present study included 772 patients diagnosed with OSMF and 760 healthy volunteers for MDA analysis. The included studies had found a statistically significant increase in serum MDA levels in OSMF patients compared with controls $(p<0.001))^{1,18,20,22,26,28,29,30,31,32,33,34,35,36,37,38}$ Similarly, other studies also observed a significant difference $p$-value $<0.01^{4}$ and $p$-value $<0.05 .{ }^{39,40} \mathrm{~A}$ few studies have also reported significantly higher salivary MDA levels in OSMF compared with healthy subjects $(p<0.001)^{41,42,43,44}$ The evaluation of tissue and mitochondrial MDA level also showed a significant rise in OSMF patients than the control group $(p<0.001) .{ }^{1.45}$ However, two included studies expressed that the increase of blood MDA level was insignificant $(p>0.05) \cdot{ }^{41,46}$

Further analysis showed a progressive increase in the serum MDA level when the clinical stage of OSMF advances. ${ }^{1,26}$ The difference in levels of MDA between the advancing stages was statistically significant $(p<0.001)^{26,31}$ and $(p<0.05)^{37,38}$ within all the clinical grades according to severity. The progressively increasing salivary malondialdehyde level was associated with a higher risk of mouth opening reduction among patients with OSMF. ${ }^{44}$ Akhlaq et $\mathrm{al}^{44}$ identified a strong negative correlation between mouth opening $(\mathrm{mm})$ and malondialdehyde $(-0.816)$. Another two studies displayed the increase in mean plasma MDA level was insignificant between clinical stages II and III. ${ }^{20,29}$ Divyambika et $\mathrm{al}^{36}$ stated that salivary lipid peroxides level was correlating with the severity of mouth opening, fibrosis, and histologic grades of OSMF. Correlation analysis of lipid peroxides levels with histological grades showed a positive correlation $(p<0.01){ }^{36}$ The authors concluded that the lipid peroxidation increases with the disease severity, reflecting the extent of tissue injury. ${ }^{5,20,26,30,39}$ Shetty et $\mathrm{al}^{1}$ reported that the tissue 


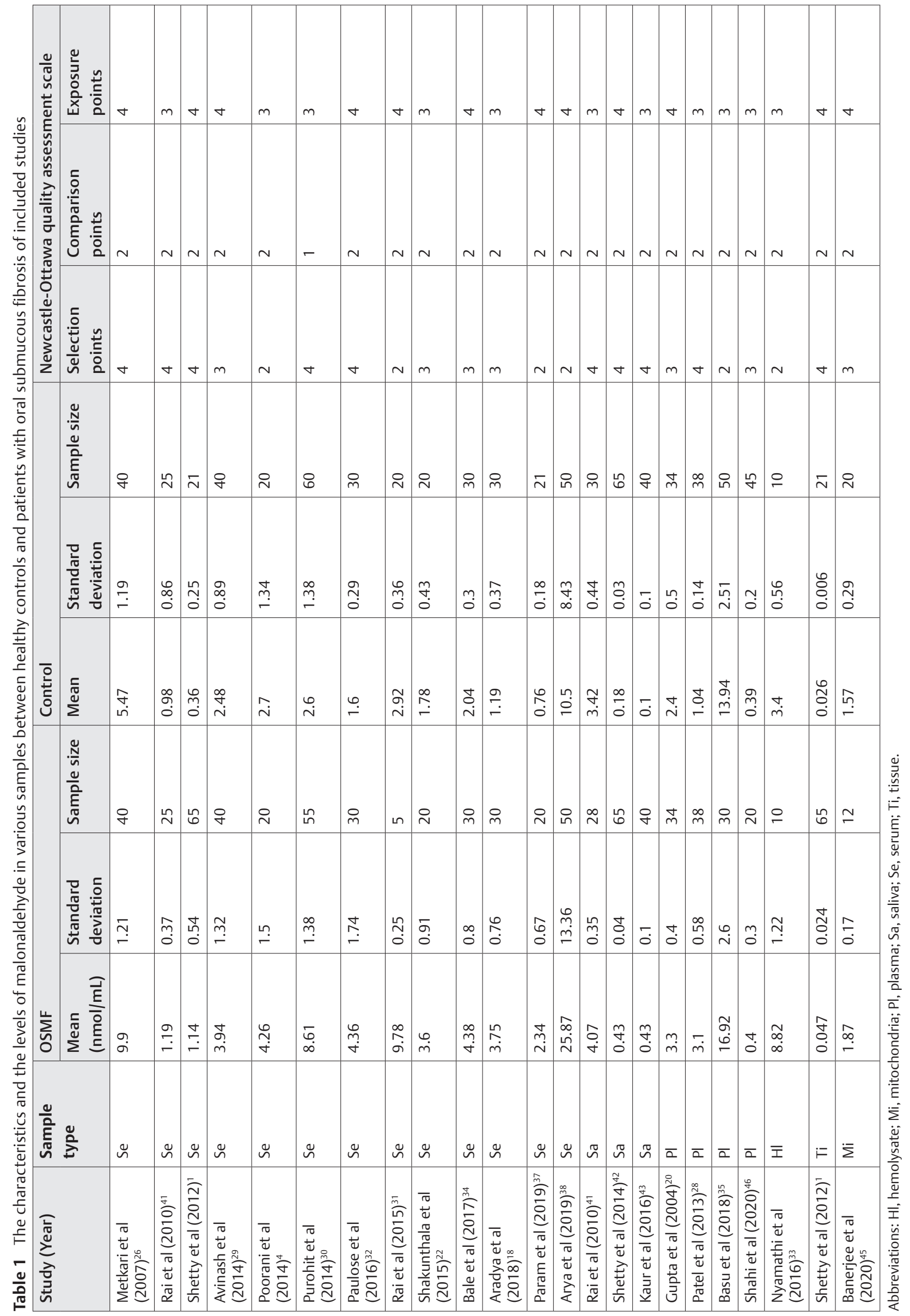




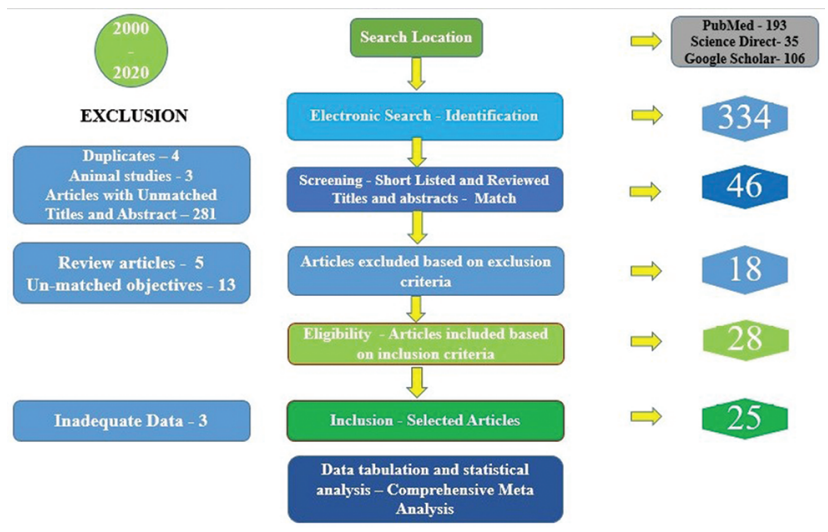

Fig. 1 Flow chart illustration study selection for the meta-analysis.

levels of MDA were consistently higher in histological grades 1 and grade 2 OSMF but decreased in grade 3 compared with controls. The resultant decrease of tissue MDA in the late advanced grade might be due to increased malondialdehyde utilization in collagen cross-linking. ${ }^{1,32,38}$ The alteration of MDA level may reflect tissue changes at a cellular level and aid in the early diagnosis of the condition. ${ }^{47}$

However, Metkari et $\mathrm{al}^{26}$ reported that the observed difference in MDA levels between different OSMF histopathological grades was statistically insignificant $(p>0.05)$. Sakunthala et $\mathrm{al}^{22}$ study did not show a significant difference in serum MDA levels concerning clinical and histopathological grading. Since OSMF may affect various parts of the oral cavity to a different extent, Metkari et $\mathrm{a}^{26}$ concluded that localized tissue histopathological examination did not reveal significant changes.

The present work displayed significantly higher lipid peroxidation in patients with OSMF. The standard difference in mean MDA concentration between OSMF and healthy subjects was estimated as $2.73 \mathrm{nmol} / \mathrm{mL}(p<0.001$, $95 \%$ CI: 2.08-3.38). Only a few studies revealed clinical and histopathological grade-wise analysis; hence, a stage or grade wise comparison was not performed. Therefore, it is vital to discover suitable biomarkers for the early diagnosis of the disease. The oxidant status assessment might help in the successful management by recognizing the earlier condition and avoiding the possible consequences of malignant transformation of OSMF.

\section{Limitations}

A limitation of the research is the relatively smaller sample size of many of the included studies in the meta-analysis. Besides, the reported studies utilized various measurement techniques and biological specimens (serum, plasma, saliva, and tissue) to assess MDA levels, resulting in ample heterogeneity between-study. Although we cannot determine with confidence that MDA levels are different between OSMF and healthy controls due to high heterogeneity, we express that the majority of the studies found significant differences statistically between OSMF patients and healthy controls.

\begin{tabular}{|c|c|c|c|c|c|c|c|c|c|c|c|}
\hline \multirow[t]{2}{*}{ Study } & \multirow[t]{2}{*}{ Subgroup } & \multicolumn{5}{|c|}{ Stetsistic for each Study } & \multicolumn{4}{|c|}{ Sid diti in means and $95 \% \mathrm{Cl}$} & \multirow{2}{*}{$\begin{array}{l}\text { Weight(Random, } \\
\text { Relative weight }\end{array}$} \\
\hline & & Std diti in & Lomer limit & Upper limit & Z-Value & p-Value & 7.00 & .350 & 0,000 & 7.00 & \\
\hline Meḱknin & Se & 3.69 & 2.97 & 4.41 & 10.04 & 0.000 & & & & - & $4.53 \mid$ \\
\hline Rai 2010 & So & 1.65 & 1.06 & 225 & 5.43 & 0.000 & & & - & & $461 j$ \\
\hline Rai 2010 & Se & 0.32 & -0.24 & 0.88 & 1.11 & 0.265 & & & & & $4.63 \mid$ \\
\hline Shenty 2014 & & 6.61 & 5.74 & 7.48 & 14.82 & 0.000 & & & & $\rightarrow$ & $4.42 i$ \\
\hline Shety 2012 & & 1.61 & 1.07 & 2.16 & 5.77 & 0.000 & & & - & & $4.64 j$ \\
\hline Shety 2012 & & 0.98 & 0.47 & 1.49 & 3.75 & 0.000 & & & + & & $4.66 \mid$ \\
\hline Avinash & Se & 1.30 & 0.81 & 1.78 & 527 & 0.000 & & & + & & 4.67 \\
\hline Poorarai & Se & 1.10 & 0.43 & 1.76 & 323 & 0.001 & & & - & & $457 i$ \\
\hline Puroht & Se & 436 & 3.68 & 5.03 & 1272 & 0.000 & & & & + & $456 \mid$ \\
\hline Paulose & Se & 2.21 & 1.57 & 2.86 & 6.75 & 0.000 & & & + & & ${ }_{4.58} \mid$ \\
\hline Rais 2015 & Se & 19.98 & 14.35 & 25.60 & 6.96 & 0.000 & & & & & $1.06 \mid$ \\
\hline Sokunntrala & Se & 256 & 1.72 & 3.39 & 6.00 & 0.000 & & & - & & $4.45 \mid$ \\
\hline Bale 2017 & Se & 3.87 & 3.02 & 4.73 & 8.85 & 0.000 & & & & $\leftarrow$ & $4.43 \mid$ \\
\hline Aredya & Se & 4.27 & 3.35 & 5.19 & 9.13 & 0.000 & & & & & 4.381 \\
\hline Param & Se & 3.25 & 2.32 & 4.18 & 6.83 & 0.000 & & & & & $4.37 \mid$ \\
\hline Aysa 2019 & Se & 1.38 & 0.94 & 1.81 & 6.19 & 0.000 & & & - & & 4.701 \\
\hline Kour 2015 & So & 3.30 & 2.63 & 397 & 960 & 0.000 & & & & & $456 \mid$ \\
\hline Gupta 2004 & PI & 1.99 & 1.41 & 257 & 6.71 & 0.000 & & & + & & 4.621 \\
\hline Bosu 2018 & P & 1.17 & 0.68 & 1.66 & 471 & 0000 & & & + & & $467 \mid$ \\
\hline Shati 2020 & P & 0.04 & -0.48 & 0.57 & 0.16 & 0.874 & & & & & 4.65 \\
\hline Nyengathi & HL L & 5.71 & 3.74 & 7.68 & 5.67 & 0.000 & & & & & 3.341 \\
\hline & Mi & 1.20 & 0.42 & 1.97 & 3.04 & 0.002 & & & - & & $4.49 \mid$ \\
\hline \multirow{2}{*}{ Patel 2013} & P & 4.88 & 3.99 & 5.78 & 10.67 & 0.000 & & & & + & 4.401 \\
\hline & & 273 & 208 & 3,38 & 8.18 & 0.000 & & & - & & \\
\hline
\end{tabular}

Fig. 2 Forest plot of the standard difference in means and 95\% confidence intervals represents differences in malondialdehyde levels of various samples between oral submucous fibrosis patients and healthy controls.

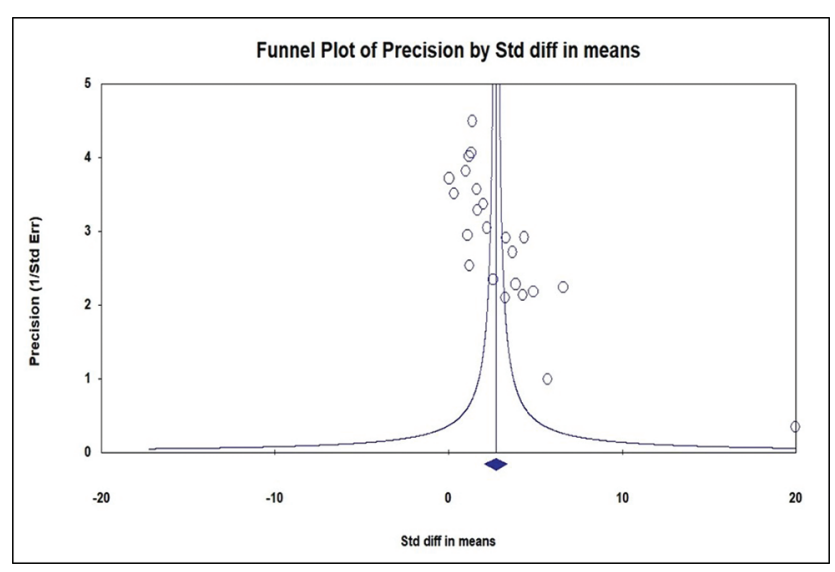

Fig. 3 Funnel plot of precision by standard difference in means.

\section{Conclusion}

The included studies in the present meta-analysis of MDA levels in OSMF showed significant differences from normal healthy controls. Despite therapeutic and diagnostic advances, the rate at which oral precancerous and cancerous lesions spread is alarming. Until now, there are no potential markers to understand the malignant transformation of potentially malignant disorders. The detection of biomarkers may also help to monitor the drug response of the disease. Additional research of large-scale studies, with equal distribution of samples among different grades of OSMF, is needed to assess the utility of MDA levels as a predictive biomarker tool with high validity and reliability.

\section{Funding}

None.

\section{Authors' Contributions}

K.M., C.K., and S.P. performed the data collection procedure. K.M., C.K., and N.J. screened the titles/abstracts and excluded studies at high risk of bias from the evidence synthesis based on prespecified exclusion criteria to avoid 
introducing bias. K.M., C.K., and S.P. independently screened each included study's full texts for eligibility. All the authors checked and discussed the relevant factors considered in each included study. The authors resolved disagreements by consensus. Finally, all the authors read and accepted the potentially included articles. K.M. wrote the initial draft of the manuscript. K.C. and N.J. participated in the manuscript revision. S.P., R.T., and L.S. reviewed the charts and final revision of the manuscript. The authors read and approved the final manuscript.

\section{Conflict of Interest}

None declared.

\section{Acknowledgments}

The authors sincerely thank Mr. Syed Imran Maktoum, Director, Kalbani Group, for his support toward the present article.

\section{References}

1 Shetty SR, Babu SG, Kumari S, Rao V, Vijay R, Karikal A. Malondialdehyde levels in oral sub mucous fibrosis: a clinicopathological and biochemical study. N Am J Med Sci 2012;4(3):125-128

2 Kanodia S, Giri VP, Giri OP, Devi MP, Garima Y. Assessment of anxiety, depression, and serum cortisol level in oral submucous fibrosis patients: a controlled clinical trial. Eur J Dent 2017;11(3):293-298

3 Rajakumar P, Saravanan R, Prabhakar R, Kumar RV, Abinesh S, Vivakanandhan U. Role of anti-oxidants in oral submucous fibrosis. J Int Oral Health 2016;8:412-414

4 Poorani R, Vezhavendhan N, Ramesh R. Vidhya Lakshmi S, Sivaramakrishnan M, Suganya R. Malondialdehyde level in oral submucous fibrosis. J Sci Dent 2014;4(2):8-13

5 D'souza D, Subhas BG, Shetty SR, Balan P. Estimation of serum malondialdehyde in potentially malignant disorders and post-antioxidant treated patients: a biochemical study. Contemp Clin Dent 2012;3(4):448-451

6 Tilakaratne WM, Klinikowski MF, Saku T, Peters TJ, Warnakulasuriya S. Oral submucous fibrosis: review on aetiology and pathogenesis. Oral Oncol 2006;42(6):561-568

7 Vincent RR, Appukuttan D, Victor DJ, Balasundaram A. Oxidative stress in chronic periodontitis patients with type II diabetes mellitus. Eur J Dent 2018;12(2):225-231

8 Canakci CF, Cicek Y, Yildirim A, Sezer U, Canakci V. Increased levels of 8-hydroxydeoxyguanosine and malondialdehyde and its relationship with antioxidant enzymes in saliva of periodontitis patients. Eur J Dent 2009;3(2):100-106

9 Irshad M, Chaudhuri PS. Oxidant-antioxidant system: role and significance in human body. Indian J Exp Biol 2002;40(11):1233-1239

10 Yegambaram M, Manivannan B, Beach TG, Halden RU. Role of environmental contaminants in the etiology of Alzheimer's disease: a review. Curr Alzheimer Res 2015;12(2):116-146

11 Anderson RA, Evans ML, Ellis GR, et al. The relationships between post-prandial lipaemia, endothelial function and oxidative stress in healthy individuals and patients with type 2 diabetes. Atherosclerosis 2001;154(2):475-483

12 Sadaksharam J. Significance of serum nitric oxide and superoxide dismutase in oral submucous fibrosis and squamous cell carcinoma: a comparative study. Contemp Clin Dent 2018;9(2):283-288

13 Matsui A, Ikeda T, Enomoto K, et al. Increased formation of oxidative DNA damage, 8 -hydroxy-2'-deoxyguanosine, in human breast cancer tissue and its relationship to GSTP1 and COMT genotypes. Cancer Lett 2000;151(1):87-95

14 Tseng SK, Chang MC, Su CY, et al. Arecoline induced cell cycle arrest, apoptosis, and cytotoxicity to human endothelial cells. Clin Oral Investig 2012;16(4):1267-1273

15 Valko M, Leibfritz D, Moncol J, Cronin MT, Mazur M, Telser J. Free radicals and antioxidants in normal physiological functions and human disease. Int J Biochem Cell Biol 2007;39(1):44-84

16 Lü JM, Lin PH, Yao Q, Chen C. Chemical and molecular mechanisms of antioxidants: experimental approaches and model systems. J Cell Mol Med 2010;14(4):840-860

17 Birben E, Sahiner UM, Sackesen C, Erzurum S, Kalayci O. Oxidative stress and antioxidant defense. World Allergy Organ J 2012;5(1):9-19

18 Aradhya CBV, Britto F, Kaikure SLHG, Paul T, Sishan U, Abdulrashid B. Shubhalakshmi. "Oxidants to assess the oxidative stress in oral submucous fibrosis. Int J CurrAdv Res 2018;7(8):14973-14975

19 Gokul S, Patil VS, Jailkhani R, Hallikeri K, Kattappagari KK. Oxidant-antioxidant status in blood and tumor tissue of oral squamous cell carcinoma patients. Oral Dis 2010;16(1):29-33

20 Gupta S, Reddy MV, Harinath BC. Role of oxidative stress and antioxidants in aetiopathogenesis and management of oral submucous fibrosis. Indian J Clin Biochem 2004;19(1):138-141

21 Agrawal S, Ambad RS. Evaluation of anti-oxidant status in oral submucous fibrosis: a hospital based study. Int J Innovat Res Med Sci 2017;2:7

22 Shakunthala GK, Annigeri RG, Arunkumar S. Role of oxidative stress in the pathogenesis of oral submucous fibrosis: A preliminary prospective study. Contemp Clin Dent 2015;6(Suppl 1):S172-S174

23 Subramanyam D, Gurunathan D, Gaayathri R. Vishnu Priya V. Comparative evaluation of salivary malondialdehyde levels as a marker of lipid peroxidation in early childhood caries. Eur J Dent 2018;12(1):67-70

24 Barrera G. Oxidative stress and lipid peroxidation products in cancer progression and therapy. ISRN Oncol 2012;2012:137289

25 Slatter DA, Paul RG, Murray M, Bailey AJ. Reactions of lipid-derived malondialdehyde with collagen. J Biol Chem 1999;274(28):19661-19669

26 Metkari S, Tupkari J, Barpande S. An estimation of serum malondialdehyde, superoxide dismutase and vitamin A in oral submucous fibrosis and its clinicopathologic correlation. J Oral Maxillofac Pathol 2007;11(1):23-27

27 Uikey AK, Hazarey VK, Vaidhya SM. Estimation of serum anti-oxidant enzymes superoxide dismutase and glutathione peroxidase in oral submucous fibrosis: a biochemical study. J Maxillofac Pathol. 2003;7:44-52

28 Patel T, Kulkarni V. A study on anti-oxidant levels in non smoke tobacco consuming oral sub mucous fibrosis (OSMF). Int J Med Res Heal Sci 2013;2:229

29 Avinash Tejasvi ML, Bangi BB, Geetha P, et al. Estimation of serum superoxide dismutase and serum malondialdehyde in oral submucous fibrosis: a clinical and biochemical study. J Cancer Res Ther 2014;10(3):722-725

30 Purohit CS. Total anti-oxidant activity - a biomarker in oral precancer patients. Int J Curr Res Rev 2014;6:21-24

31 Rai S, Sharma A, Ranjan V, Misra D, Panjwani S. Estimation of serum anti-oxidant enzymes in histopathological grades of oral leukoplakia, oral submucous fibrosis, and oral cancer: a clinicopathologic study. J Indian Acad Oral Med Radiol 2015;27:349

32 Paulose S, Rangdhol V, Ramesh R, Jeelani SA, Brooklyin S. Estimation of serum malondialdehyde and assessment of DNA damage using comet assay in patients with oral submucous fibrosis. J Investig Clin Dent 2016;7(3):286-293

33 Nyamati SB, Annapoorna HB, Tripathi J, Sinha N, Roy S, Agrawal R. Evaluation of serum anti-oxidant enzymes 
in oral submucous fibrosis and oral squamous cell carcinoma: a clinical and biochemical study. J Adv Med Dent Sci Res 2016;4:83-87

34 Bale R, Kattappagari KK, Vidya D, Vuddandi S, Gummalla C, Baddam VRR. Oral submucous fibrosis: a quantitative assessment of serum malondialdehyde, superoxide dismutase and correlation with clinical staging. J Oral Maxillofac Pathol 2017;21(1):41-45

35 Basu S, Guhan VN. Enzymatic and non-enzymatic anti-oxidants changes in pre-cancerous and cancerous lesions of oral cavity. Med Pulse Int J Biochem 2018;5:54-58

36 Divyambika CV, Sathasivasubramanian S, Vani G, Vanishree AJ, Malathi N. Correlation of clinical and histopathological grades in oral submucous fibrosis patients with oxidative stress markers in saliva. Indian J Clin Biochem 2018;33(3):348-355

37 Param K, Kanchan M, Manish P, Satish K. Assessment of serum paraoxonase-1 enzyme activity, malondialdehyde and vitamin-C in oral premalignancies. Int J Clin Biomed Res 2018;4:21-26

38 Arya H, Ganvir SM, Begde DN, Passi AD. Comparative evaluation of serum malondialdehyde (MDA) level in oral submucous fibrosis and oral squamous cell carcinoma. J Clin Diagn Res 2019;13(6):ZC27-ZC31

39 Bathi RJ, Rao R, Mutalik S. GST null genotype and antioxidants: risk indicators for oral pre-cancer and cancer. Indian J Dent Res 2009;20(3):298-303
40 Oswal RG, Rao KN, Mall P, Grover I. Evaluation of serum antioxidant enzymes in oral submucous fibrosis and oral squamous cell carcinoma- a clinical and biochemical study. Eur J Mol Clin Med 2020;7(8):3205-3209

41 Rai B, Kaur J, Jacobs R, Singh J. Possible action mechanism for curcumin in pre-cancerous lesions based on serum and salivary markers of oxidative stress. J Oral Sci 2010;52(2):251-256

42 Shetty SR, Babu S, Kumari S, Shetty P, Hegde S, Castelino R. Status of salivary lipid peroxidation in oral cancer and precancer. Indian J Med Paediatr Oncol 2014;35(2):156-158

43 Kaur J, Politis C, Jacobs R. Salivary 8-hydroxy-2-deoxyguanosine, malondialdehyde, vitamin C, and vitamin $\mathrm{E}$ in oral pre-cancer and cancer: diagnostic value and free radical mechanism of action. Clin Oral Investig 2016;20(2):315-319

44 Akhlaq H, Mehmood N, Azfar M. Increase level of salivary malondialdehyde is associated with decrease mouth opening in stage-I oral submucous fibrosis. PakJ Surg 2017;33(3):195-200

45 Banerjee S, Mukherjee S, Mitra S, Singhal P. Comparative evaluation of mitochondrial antioxidants in oral potentially malignant disorders. Kurume Med J 2020;66(1):15-27

46 Shahi Y, Samadi FM, Mukherjee S. Plasma lipid peroxidation and anti-oxidant status in patients with oral pre-cancerous lesions and oral cancer. Oral Sci Int 2020;0:1-8

47 Gupta RK, Patel AK, Shah N, et al. Oxidative stress and antioxidants in disease and cancer: a review. Asian Pac J Cancer Prev 2014;15(11):4405-4409 